\title{
Mining Frequent Patterns in Evolving Graphs
}

\author{
Cigdem Aslay , Muhammad Anis Uddin Nasir ${ }^{\star}$, \\ Gianmarco De Francisci Morales ${ }^{\circ}$, Aristides Gionis $\sharp$ \\ ${ }^{\ddagger}$ ISI Foundation ${ }^{\star}$ Royal Institute of Technology ${ }^{\circledR}$ Qatar Computing Research Institute ${ }^{\sharp}$ Aalto University \\ cigdem.aslay@isi.it, anisu@kth.se,gdfm@acm.org, aristides.gionis@aalto.fi
}

\begin{abstract}
Given a labeled graph, the frequent-subgraph mining (FSM) problem asks to find all the $k$-vertex subgraphs that appear with frequency greater than a given threshold. FSM has numerous applications ranging from biology to network science, as it provides a compact summary of the characteristics of the graph. However, the task is challenging, even more so for evolving graphs due to the streaming nature of the input and the exponential time complexity of the problem. In this paper, we initiate the study of approximate FSM problem in both incremental and fully-dynamic streaming settings, where arbitrary edges can be added or removed from the graph. For each streaming setting, we propose algorithms that can extract a high-quality approximation of the frequent $k$-vertex subgraphs for a given threshold, at any given time instance, with high probability. In contrast to the existing state-of-the-art solutions that require iterating over the entire set of subgraphs for any update, our algorithms operate by maintaining a uniform sample of $k$-vertex subgraphs with optimized neighborhood-exploration procedures local to the updates. We provide theoretical analysis of the proposed algorithms and emprically demonstrate that the proposed algorithms generate high-quality results compared to baselines.
\end{abstract}

\section{INTRODUCTION}

Frequent-subgraph mining (FSM) is a fundamental graph-mining task with applications in various disciplines, including bioinformatics, security, and social sciences. The goal of FSM is to find subgraph patterns of interest that are frequent in a given graph. Such subgraphs might be indicative of an important protein interaction, a possible intrusion, or a common social norm. FSM also finds applications in graph classification and indexing.

Existing algorithms for subgraph mining are not scalable to large graphs that arise, for instance, in social domains. In addition, these graphs are usually produced as a result of a dynamic process, hence are subject to continuous changes. For example, in social networks new edges are added as a result of the interactions of their users, and the graph structure is in continuous flux. Whenever the graph changes, e.g., by adding or removing an edge, a large number of new subgraphs can be created, and existing subgraphs can be modified or destroyed. Keeping track of all the possible changes in the graph is subject to combinatorial explosion, thus, is highly challenging.

Permission to make digital or hard copies of all or part of this work for personal or classroom use is granted without fee provided that copies are not made or distributed for profit or commercial advantage and that copies bear this notice and the full citation on the first page. Copyrights for components of this work owned by others than ACM must be honored. Abstracting with credit is permitted. To copy otherwise, or republish, to post on servers or to redistribute to lists, requires prior specific permission and/or a fee. Request permissions from permissions@acm.org.

Conference'17, Washington, DC, USA

(C) 2016 ACM. 978-x-xxxx-xxxx-x/YY/MM...\$15.00

DOI: $10.1145 /$ nnnnnnn.nnnnnnn
In this paper we address the problem of mining frequent subgraphs in an evolving graph, which is represented as a stream of edge updates - additions or deletions. Only a few existing works consider a similar setting [3, 29].

Bifet et al. [3] deal with a transactional setting where the input is a stream of small graphs. Their setting is similar to the one considered by frequent-itemset mining, so many of the existing results can be reused. Conversely, in our case, there is a single graph that is continuously evolving. Ray et al. [29] consider a scenario similar to the one we study in this paper. They consider a single large graph with continuous updates, although they only allow incremental updates (edge addition) rather than the fully-dynamic updates we consider (edge addition and deletion). Moreover, their approach is a simple heuristic that does not provide any correctness guarantee. Our approach, instead, is able to provably find the frequent subgraphs in a fully dynamic graph stream.

This paper is the first to study the frequent-subgraph mining problem on a fully dynamic evolving graph. We propose a principled sampling scheme for subgraphs and provide theoretical justifications for its accuracy. Differently from previous work on sampling from graph streams, our method relies on sampling subgraphs rather than sampling edges. This choice enables sampling any kind of subgraph of the same size with equal probability, and thus simplifies dramatically the design of the frequency estimators. We maintain a uniform sample of subgraphs of the graph via reservoir sampling, which in turn allows us to estimate the frequency of different patterns. To handle deletions in the stream, we employ an adapted version of random pairing [12]. Finally, to increase the efficiency of our sampling procedure during the exploration of the local neighborhood of updated edges, we employ an adaptation of the "skip optimization," proposed by Vitter [35] for reservoir sampling and by Gemulla et al. [13] for random pairing.

Concretely, our main contributions are the following:

- We are the first to study the problem of frequent-subgraph mining on evolving labeled graphs.

- We propose a new subgraph-based sampling scheme.

- We show how to use random pairing to handle deletions.

- We describe how to implement neighborhood exploration efficiently via "skip optimization."

- We provide theoretical analysis and guarantees on the accuracy of the algorithm.

\section{PROBLEM DEFINITION}

We consider graphs with vertex and edge labels. We model dynamic graphs as a sequence of edge additions and deletions.

We assume monitoring a graph that changes over time. For any time $t \geq 0$, we let $G^{t}=\left(V^{t}, E^{t}\right)$ be the graph that has been observed up to and including time $t$, where $V^{t}$ represents the set of vertices and $E^{t}$ represents the set of edges. We assume that vertices and 
edges have labels, and we write $L$ and $Q$ for the sets of labels of vertices and edges, respectively. For each vertex $v \in V^{t}$ we denote its label by $\ell_{v} \in L$, and similarly, for each edge $e=(u, v) \in E^{t}$ we denote its label by $q_{e} \in Q$. Initially, at time $t=0$, we have $V^{0}=E^{0}=\emptyset$. For any $t \geq 0$, at time $t+1$ we receive an update tuple $\langle\mathbf{o}, e, q\rangle$ from a stream, where $\mathbf{o} \in\{+,-\}$ represents an update operation, addition or deletion, $e=(u, v)$ is a pair of vertices, and $q \in Q$ is an edge label. The graph $G^{t+1}=\left(V^{t+1}, E^{t+1}\right)$ is obtained by adding a new edge or deleting an existing edge as follows:

$$
E^{t+1}=\left\{\begin{array}{ll}
E^{t} \cup\{e\} \text { and } q_{e}=q & \text { if } \mathbf{o}=+ \\
E^{t} \backslash\{e\} & \text { if } \mathbf{o}=-
\end{array} .\right.
$$

Additions and deletions of vertices are treated similarly. Furthermore, we assume that when adding an edge $e=(u, v)$, the vertices $u$ and $v$ are added in the graph too, if they are not present at time $t$. Similarly, when deleting a vertex, we assume that all incident edges are deleted too, prior to the vertex deletion. For simplicity of exposition in the rest of the paper we discuss only edge additions and deletions - vertex operations can be handled rather easily.

We use $n_{t}=\left|V^{t}\right|$ and $m_{t}=\left|E^{t}\right|$ to refer to the number of vertices and edges, respectively, at time $t$. In this work, we considered simple, connected, and undirected graphs. The neighborhood of a vertex $u \in V^{t}$ at time $t$ is defined as $N_{u}^{t}=\left\{v \mid(u, v) \in E^{t}\right\}$, and its degree as $d_{u}^{t}=\left|N_{u}^{t}\right|$. Similarly, the $h$-hop neighborhood of $u$ at time $t$ is denoted as $N_{u, h}^{t}$, and indicates the set of the vertices that can be reached from $u$ in $h$ steps by following the edges $E^{t}$. To simplify the notation, we omit to specify the dependency on $t$ when it is obvious from the context.

For any graph $G=(V, E)$ and a subset of vertices $S \subseteq V$, we say that $G_{S}=(S, E(S))$ is an induced subgraph of $G$ if for all pairs of vertices $u, v \in S$ it is $(u, v) \in E(S)$ if and only if $(u, v) \in E$. We define $C^{k}$ to be the set of all induced subgraphs with $k$ vertices in $G$. All subgraphs considered in this paper are induced subgraphs, unless stated otherwise.

We say that two subgraphs of $G$, denoted by $G_{S} \in C^{k}$ and $G_{T} \in C^{k}$ are isomorphic if there exists a bijection $I: S \rightarrow T$ such that $(u, v) \in E(S)$ if and only if $(I(u), I(v)) \in E(T)$ and the mapping $I$ preserves the vertex and edge labels, i.e., $\ell_{u}=\ell_{I(u)}$ and $q_{(u, v)}=q_{(I(u), I(v))}$, for all $u \in S$ and for all $(u, v) \in E(S)$. We write $G_{S} \simeq G_{T}$ to denote that $G_{S}$ and $G_{T}$ are isomorphic.

The isomorphism relation $\simeq$ partitions the set of subgraphs $C^{k}$ into $T_{k}$ equivalence classes $C_{1}^{k}, \cdots, C_{T_{k}}^{k}$. Each equivalence class $C_{i}^{k}$ is called a subgraph pattern.

We define the support set $\sigma\left(G_{S}\right)$ of any $k$-vertex subgraph $G_{S} \in$ $C^{k}$ as the number of $k$-vertex subgraphs of $G$ that are isomorphic to $G_{S}$, i.e., $\sigma\left(G_{S}\right)=\left|C_{i}^{k}\right|$, where $G_{S} \in C_{i}^{k}$. We then define the frequency $f\left(G_{S}\right)$ of a subgraph $G_{S}$ as the fraction of $k$-vertex subgraphs of $G$ that are isomorphic to $G_{S}$, i.e., $f\left(G_{S}\right)=\sigma\left(G_{S}\right) /\left|C^{k}\right|$.

Next we define the problem of mining frequent $k$-vertex subgraphs. Given a graph $G=(V, E, L, Q)$ and a frequency threshold $\tau \in(0,1]$, the set $\mathcal{F}_{\tau}^{k} \subseteq C^{k}$ of frequent $k$-vertex subgraphs of $G$ with respect to $\tau$ is the collection of all $k$-vertex subgraphs with frequency at least $\tau$, that is

$$
\mathcal{F}_{\tau}^{k}=\left\{G_{S} \mid G_{S} \in C^{k} \text { and } f\left(G_{S}\right) \geq \tau\right\} .
$$

Problem 2.1. Given a graph $G=(V, E, L, Q)$, an integer $k$, and a frequency threshold $\tau$, find the collection $\mathcal{F}_{\tau}^{k}$ of frequent $k$-vertex subgraphs of $G$.

Let $p_{i}=\left|C_{i}^{k}\right| /\left|C^{k}\right|$ denote the frequency of isomorphism class $i$, with $i=1, \ldots, T_{k}$. The problem of finding the frequent $k$-vertex subgraphs requires finding all isomorphism classes $C_{i}^{k}$ with $p_{i} \geq \tau$. Hence, we equivalently have

$$
\mathcal{F}_{\tau}^{k}=\bigcup_{i \in\left[1, T_{k}\right]}\left\{G_{S} \mid G_{S} \in C_{i}^{k} \text { and } p_{i} \geq \tau\right\} .
$$

In this paper, our aim is to find an approximation to the collection $\mathcal{F}_{\tau}^{k}$ by efficiently estimating $p_{i}$, from a uniform sample $\mathcal{S}$ of $C^{k}$. We say that a subset $\mathcal{S} \subseteq C^{k}$, with $|\mathcal{S}|=M$, is a uniform sample of size $M$ from $C^{k}$ if the probability of sampling $\mathcal{S}$ is equal to the probability of sampling any $\mathcal{S}^{\prime} \subseteq C^{k}$ with $\left|\mathcal{S}^{\prime}\right|=M$, i.e., all samples of the same size are equally likely to be produced.

Formally, we want to find an $(\epsilon, \delta)$-approximation to $\mathcal{F}_{\tau}^{k}$, denoted by $\tilde{\mathcal{F}}_{\tau}^{k}(\epsilon, \delta)$ such that

$$
\tilde{\mathcal{F}}_{\tau}^{k}(\epsilon, \delta)=\bigcup_{i \in\left[1, T_{k}\right]}\left\{G_{S}\left|G_{S} \in C_{i}^{k} \cap \mathcal{S},\right| \hat{p}_{i}-p_{i} \mid \leq \epsilon / 2, p_{i} \geq \tau\right\},
$$

where $\hat{p}_{i}$ is the estimation of $p_{i}$ such that $\left|\hat{p_{i}}-p_{i}\right| \leq \epsilon / 2$ holds with probability at least $1-\delta$. In practice, the collection $\tilde{\mathcal{F}}_{\tau}^{k}(\epsilon, \delta)$ of approximate frequent patterns is computed from a sample $\mathcal{S} \subseteq C^{k}$.

The problem of approximate frequent subgraph mining can now be formulated as follows.

Problem 2.2. Given a graph $G=(V, E, L, Q)$, a frequency threshold $\tau$, a small integer $k$, and constants $0<\epsilon, \delta<1$, find the collection $\tilde{\mathcal{F}}_{\tau}^{k}(\epsilon, \delta)$ that is an $(\epsilon, \delta)$-approximation to $\mathcal{F}_{\tau}^{k}$.

We focus on the dynamic case with vertex and edge additions and insertions. As discussed above, at each time $t$ we consider the $G^{t}=\left(V^{t}, E^{t}\right)$ that results from all vertex and edge operations. Our goal is to maintain the approximate collection of frequent subgraphs $\tilde{\mathcal{F}}_{\tau}^{k}(\epsilon, \delta)$ at each time $t$ without having to recompute it from scratch after each addition or deletion.

In the following problem definition we assume that vertex/edge labels are specified when a vertex/edge is added in the graph stream and they do not change afterwards. We make this assumption without loss of generality, as a vertex/edge label change can be simulated by a vertex/edge deletion followed by an addition of the same vertex/edge with different label.

Problem 2.3. Given an evolving graph $G^{t}=\left(V^{t}, E^{t}, L, Q\right), a$ frequency threshold $\tau$, a small integer $k$, and constants $0<\epsilon, \delta<1$, maintain an approximate collection of frequent subgraphs $\tilde{\mathcal{F}}_{\tau}^{k}(\epsilon, \delta)$ at each time $t$.

\section{ALGORITHMS}

This section describes the proposed algorithms, which are based on subgraph sampling. We present two algorithms, both of which are based on two components: a reservoir of samples and an exploration procedure. The goal of the reservoir is to capture the changes to already sampled connected $k$-subgraphs. ${ }^{1}$ The goal of the exploration procedure is to include newly (dis)connected $k$ subgraphs into the sample. This separation of concern allows the

${ }^{1}$ Hereafter, we simply refer to a $k$-vertex induced subgraph as $k$-subgraph. 
algorithm to minimize the amount of work per sample, e.g., by avoiding computation of expensive minimum DFS codes for the corresponding patterns [38].

The base algorithm requires to enumerate, at each time $t$, every newly (dis)connected $k$-subgraph at least once, by performing a neighborhood exploration of the updated edge. We show how to improve this algorithm by avoiding to materialize all the subgraphs via a skip optimization. This optimization enables picking subgraphs into sample without having to list them all. We also propose an additional heuristic to speed up the neighborhood exploration. We provide an efficient implementation for the case $k=3$, and describe how it generalizes to values $k>3$ (although not as efficiently).

\subsection{Incremental streams}

We begin by describing our algorithm for maintaining a uniform sample $\mathcal{S}$ of fixed-size $M$ of $k$-subgraphs of $G^{t}$ for incremental streams (only edge addition). The algorithm relies on reservoir sampling [35] to ensure the uniformity of the sample $\mathcal{S}$.

The addition of an edge $(u, v) \notin E^{t-1}$ at time $t$ affects only the subgraphs in the local neighborhoods up to $N_{u, h}^{t-1}$ and $N_{v, j}^{t-1}$, where $h+j=k-2$, i.e., all the connected $k$-subgraphs that contain $u$, $v$, and $h+j$ additional nodes from their neighborhoods, for all admissible values of $h, j>0$. Therefore, a uniform sample $\mathcal{S}$ of subgraphs can be maintained by iterating through the subgraphs in the neighborhood of the newly inserted edge. In particular, consider the addition of an edge $(u, v)$ at time $t$. Let $H \subseteq N_{u, h}^{t-1} \cup N_{v, j}^{t-1}$ be a subset of vertices, for some $h$ and $j$, such that $h+j=k-2,\{u, v\} \in H$, $|H|=k$, and $\{u, v\} \in H$. There are two possible cases: $(i)$ if $H$ is connected in $G^{t-1}$, a modified subgraph $H^{\prime}=H \cup\{(u, v)\}$ is formed in $G^{t}$; (ii) if $H$ is not connected in $G^{t-1}$, and $H^{\prime}=H \cup\{(u, v)\}$ is connected in $G^{t}, H^{\prime}$ is a newly formed connected $k$-subgraph in $G^{t}$.

Example for $k=3$. Assume an edge $(u, v)$ arrives at time $t$. For case (i) to hold, there should be some $w \in N_{u}^{t-1} \cap N_{v}^{t-1}$ for which the edge $(u, v)$ closes the wedge $\wedge=\{(u, w),(w, v)\}$ at $G^{t-1}$, forming a new triangle $\Delta=\wedge \cup\{(u, v)\}$ in $G^{t}$. For case (ii) to hold, there must be some $w \in N_{u}^{t-1}$ (or $w \in N_{v}^{t-1}$ ), for which a new wedge $\{(u, v),(u, w)\}$ (respectively, $\{(u, v),(v, w)\})$ is formed in $G^{t}$.

When a modified subgraph $H^{\prime}$ is formed in $G^{t}$, if the previously connected subgraph $H=H^{\prime} \backslash\{(u, v)\}$ is present in $\mathcal{S}$, we update the sample by substituting $H^{\prime}$ with $H$. Otherwise, we ignore the modified subgraph. Given that the elements in the sample are induced connected subgraphs, this operation is equivalent to maintaining the sample up-to-date.

Conversely, when a new connected $k$-subgraph $H^{\prime}$ is formed in $G^{t}$, we can be sure that it appears at time $t$ for the first time. Therefore, we use the standard reservoir sampling algorithm as follows: If $|\mathcal{S}|<M$, we directly add the new subgraph $H^{\prime}$ to the sample $\mathcal{S}$. Otherwise, if $|\mathcal{S}|=M$, we remove a randomly selected subgraph in $\mathcal{S}$ and insert the new one $H^{\prime}$ with probability $M / N$, where $M$ is the upper bound on the sample size and $N$ is the total number of (valid) $k$-subgraphs encountered since $t=0{ }^{2}$ The modification of existing subgraphs in $G^{t}$ (i.e., case $(i)$ ) does not

\footnotetext{
${ }^{2}$ Note that the addition of an edge $(u, v)$ translates to partially-dynamic $k$-subgraph streams in which the $k$-subgraphs are subject to addition and deletion operations, while $k$-cliques are subject to addition-only operations. Thus, we can impose, without loss of generality, an order of operation during the exploration of the neighborhood of the inserted edge.
}

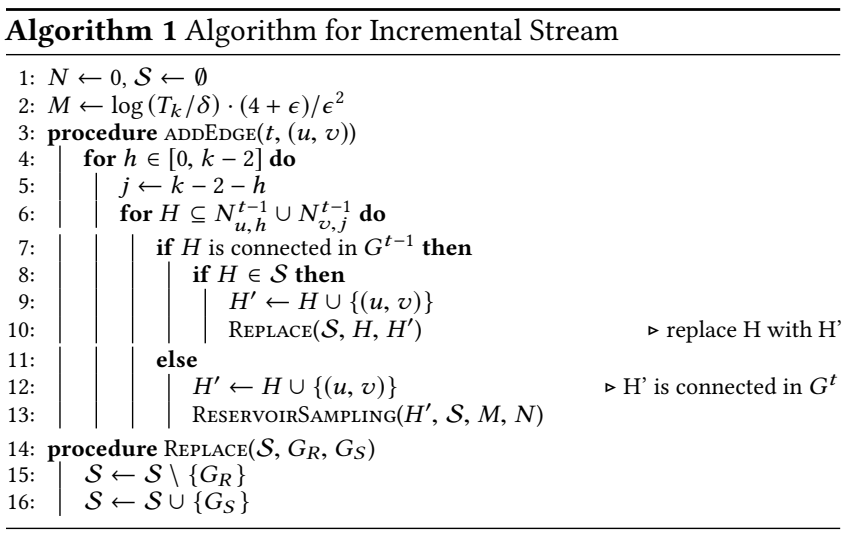

affect $N$, since, by definition, they replace the previous subgraphs which were already present in $G^{t-1}$. Therefore, the only increase in the number $N$ of subgraphs occurs in the case of new connected $k$-subgraph formations in $G^{t}$ (i.e., case $(i i)$ ).

Algorithm 1 shows the pseudocode for incremental streams. Next, we show that the sample $\mathcal{S}$ maintained by Algorithm 1 is uniform at any given time $t$.

CLAIM 3.1. Algorithm 1 ensures the uniformity of the sample $\mathcal{S}$ at any time $t$.

Proof. To show that $\mathcal{S}$ is uniform, we need to consider two cases: ( $i$ ) the inserted edge modifies an existing $k$-subgraph; (ii) the inserted edge forms a newly connected $k$-subgraph.

For the case of new subgraph formation, the uniformity property directly holds as it leverages the standard reservoir sampling algorithm. Now, we show that the uniformity property holds when a subgraph is modified.

Assume the edge $(u, v) \notin E^{t-1}$ is inserted at time $t$ and let $H$ denote the invalidated subgraph that is modified as $H^{\prime}=H \cup$ $\{(u, v)\}$ at time $t$. Let $\mathcal{S}^{\prime}$ denote the sample after the invalidation of $H$ and the formation of $H^{\prime}$. For the sample to be truly uniform, the probability that $H^{\prime} \in \mathcal{S}^{\prime}$ should be equal to $M / N$, conditioned on $\mathcal{S}=M<N$ (conditioning on $\mathcal{S}=N<M$ is trivial since every $k$-subgraph of $G^{t}$ would then be deterministically included in $\mathcal{S}^{\prime}$ ). Now, given that $\operatorname{Pr}(H \in \mathcal{S})=M / N$, we have that

$$
\begin{aligned}
\operatorname{Pr}\left(H^{\prime} \in \mathcal{S}^{\prime}\right) & =\operatorname{Pr}\left(H \in \mathcal{S}, H^{\prime} \in \mathcal{S}^{\prime}\right)+\operatorname{Pr}\left(H \notin \mathcal{S}, H^{\prime} \in \mathcal{S}^{\prime}\right) \\
& =\frac{M}{N} \cdot 1+\left(1-\frac{M}{N}\right) \cdot 0=\frac{M}{N},
\end{aligned}
$$

hence uniformity is preserved.

\subsection{Fully dynamic streams}

In this section we describe our algorithm for maintaining a uniform sample $\mathcal{S}$ of fixed size $M$ for fully-dynamic edge streams (edge insertions and deletions). Our algorithm relies on random pairing (RP) [13], a sampling scheme that extends traditional reservoir sampling for evolving data streams, in which elements are subject to both addition and deletion operations. 


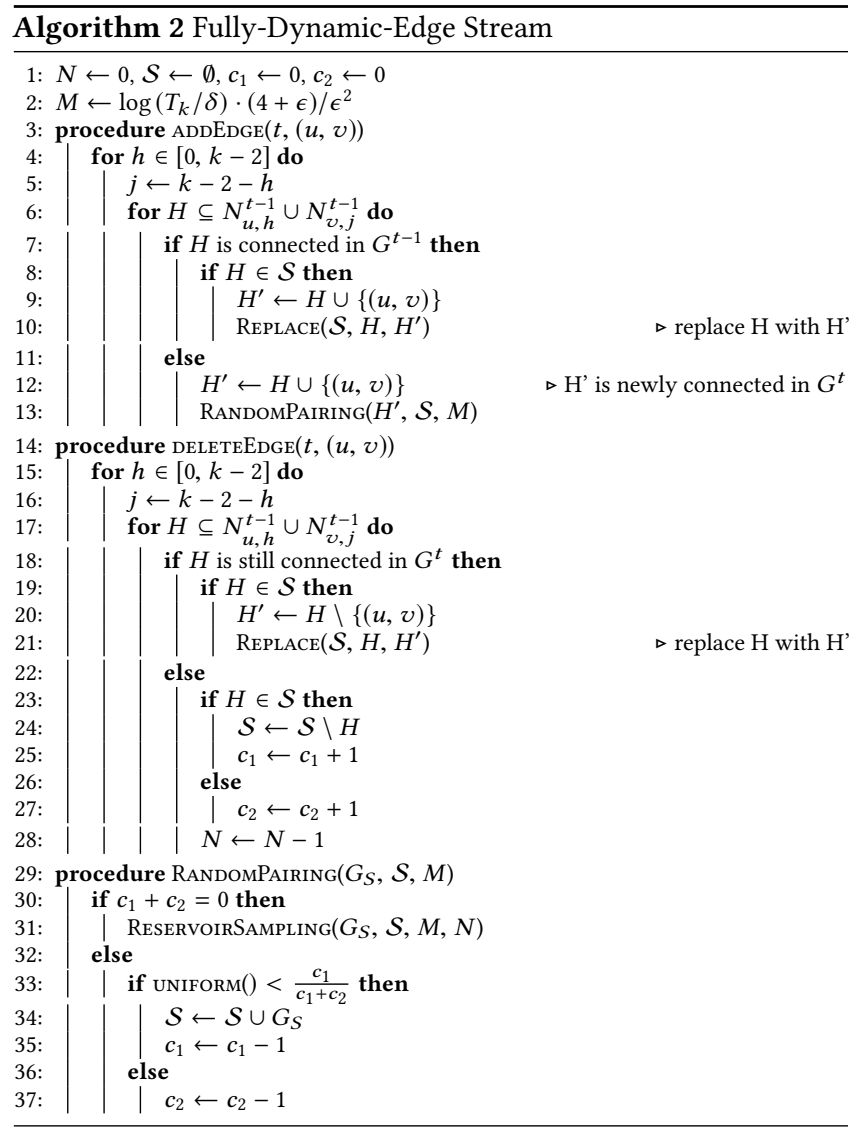

We first give a brief background on the RP scheme. In RP, the uniformity of the sample is guaranteed by randomly pairing an inserted element with an uncompensated "partner" deletion, without necessarily keeping the identity of the partner. At any time, there can be 0 or more uncompensated deletions, denoted by $d$, which is equal to the difference between the cumulative number of insertions and the cumulative number of deletions. The RP algorithm maintains $(i)$ a counter $c_{1}$ that records the number of uncompensated deletions in which the deleted element was in the sample, (ii) a counter $c_{2}$ that records the number of uncompensated deletions in which the deleted element was not in the sample, hence, $d=c_{1}+c_{2}$. When $d=0$, i.e., when there are no uncompensated deletions, inserted elements are processed as in standard reservoir-sampling. When $d>0$, the algorithm flips a coin at each inserted element and includes it in the sample with probability $c_{1} /\left(c_{1}+c_{2}\right)$, otherwise it excludes it from the sample.

Next, we describe our adaptation of the RP scheme for fullydynamic edge streams, which translate to fully-dynamic $k$-subgraph streams. First, remember that the incremental stream translates to an incremental $k$-subgraph stream, in which connected $k$-subgraphs are only added (the first time they are created) or modified (when new induced edges arrive).

In the case of fully-dynamic edge streams, the $k$-connected subgraph stream is also subject to addition and deletion operations, as we explain next.
The events of interest regarding the addition of an edge has been discussed extensively in the previous section hence we do not repeat it here. Consider the deletion of an edge $(u, v) \in E^{t-1}$ at time $t$, and a subgraph $H \subseteq N_{u, h}^{t-1} \cup N_{v, j}^{t-1}$ in $G^{t-1}$, with $h+j=k-2$. The effect of the edge deletion is the following: either $(i)$ the vertices of $H$ remain connected, hence, $H$ is replaced by a new subgraph $H^{\prime}$ in $G^{t}$; or (ii) $H$ gets disconnected, hence $H$ does not exist in $G^{t}$.

The first case corresponds to a modification of an existing connected $k$-subgraph. As such, it does not cause an addition or deletion in the subgraph stream.

Example for $\mathbf{k}=3$. In the case a triangle $\Delta$ in $G^{t-1}$ that contains an edge $(u, v)$ deleted at time $t$, if $\Delta \in \mathcal{S}$, we modify the corresponding induced subgraph into a subgraph $\wedge=\Delta \backslash\{(u, v)\}$.

The second case corresponds to a deletion of a subgraph in the stream. To handle this case, our sampling strategy follows the RP scheme. In the case that a subgraph $H$ in $G^{t-1}$ is deleted due to the deletion of edge $(u, v)$ at time $t$, if $H \in \mathcal{S}$, we increment the counter $c_{1}$, otherwise we increment the counter $c_{2}$. In the case that a new subgraph $H^{\prime}$ is formed in $G^{t}$ due to the addition of edge $(u, v)$ at time $t$, we include it in $\mathcal{S}$ with probability $c_{1} /\left(c_{1}+c_{2}\right)$. The approach is shown in Algorithm 2. Next, we show that the sample $\mathcal{S}$ maintained by Algorithm 2 is uniform at any given time $t$.

CLAIM 3.2. Algorithm 2 ensures the uniformity of the sample $\mathcal{S}$ at any time $t$.

Proof. To show that $\mathcal{S}$ is uniform, we need to consider four cases: ( $i$ ) added edge forms a newly connected subgraph; (ii) deleted edge disconnects a subgraph; (iii) added edge modifies an existing a subgraph; (iv) deleted edge modifies an existing a subgraph. Given that the additions in case ( $i$ ) and deletions in case ( $i i)$ are handled by $\mathrm{RP}$, the uniformity of the sample for these two operations directly follows from the correctness of RP. Now we show the correctness in cases $(i i i)$ and $(i v)$. Assume the edge $(u, v) \in E^{t-1}$ is deleted (resp. added) at time $t$. Let $H^{\prime}$ denote the new subgraph due to the deletion (addition) of the edge, so that $H^{\prime}=H \backslash\{(u, v)\}$ (resp. $H^{\prime}=$ $H \cup\{(u, v)\})$. Let $\mathcal{S}^{\prime}$ denote the sample after the invalidation of $H$ and the formation of $H^{\prime}$. Recall that $N$ remains unchanged since $H^{\prime}$ replaces $H$ in $G^{t}$. Given that the random pairing scheme guarantees uniformity of the sample at each time instance independently from the current value of $d$ [12], we have $\operatorname{Pr}(H \in \mathcal{S})=|\mathcal{S}| / N$. For the sample to be truly uniform, the probability that $H^{\prime} \in \mathcal{S}^{\prime}$ should also be equal to $|\mathcal{S}| / N$ since the values of both $N$ and $\mathcal{S}$ remain unchanged as we either replace $H$ with $H^{\prime}$ in $\mathcal{S}$ or we ignore $H^{\prime}$ if $H \notin \mathcal{S}$, hence $|\mathcal{S}|$ remains unchanged. Thus, we have,

$$
\begin{aligned}
\operatorname{Pr}\left(H^{\prime} \in \mathcal{S}^{\prime}\right) & =\operatorname{Pr}(H \in \mathcal{S}) \cdot \operatorname{Pr}\left(H^{\prime} \in \mathcal{S}^{\prime} \mid H \in \mathcal{S}\right) \\
& +\operatorname{Pr}(H \notin \mathcal{S}) \cdot \operatorname{Pr}\left(H^{\prime} \in \mathcal{S}^{\prime} \mid H \notin \mathcal{S}\right) \\
& =\frac{|\mathcal{S}|}{N} \cdot 1+\left(1-\frac{|\mathcal{S}|}{N}\right) \cdot 0=\frac{|\mathcal{S}|}{N},
\end{aligned}
$$

hence uniformity is preserved.

\subsection{Skip optimization}

The basic algorithm for incremental streams we described requires to process each subgraph $H \subseteq N_{u, h}^{t-1} \cup N_{v, j}^{t-1}$, for all admissible values of $h, j>0$ s.t. $h+j=k-2$, to identify among them the 

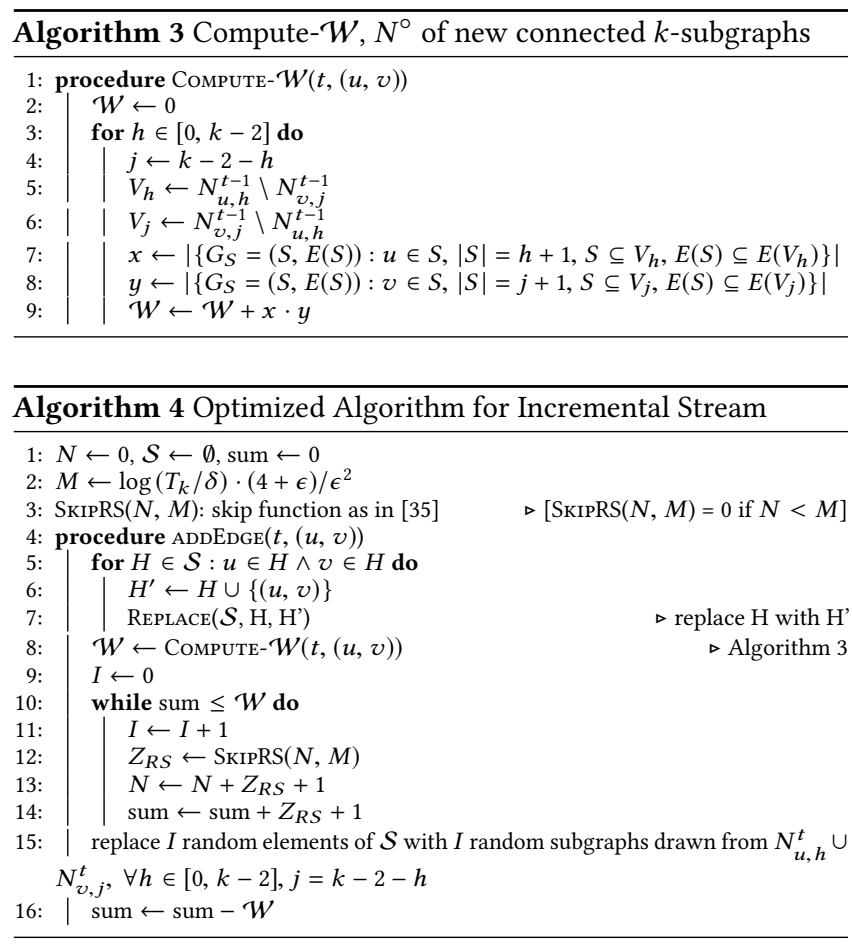

newly created $k$-subgraphs. All these new subgraphs are then provided as input to the standard reservoir sampling algorithm that needs to generate random numbers for each. To reduce the cost of traversing the local neighborhood and generating random numbers for each new subgraph, we exploit Vitter's acceptancerejection algorithm [35] for standard reservoir sampling as follows: let $Z_{R S}$ be the random variable that denotes the number of rejected subgraphs after the last time a subgraph was inserted to the sample $\mathcal{S}$. Then, the probability that the next $z$ new subgraphs will not be accepted in $\mathcal{S}$ is given by:

$$
\operatorname{Pr}\left[Z_{R S}=z\right]=\frac{M}{N+z+1} \prod_{z^{\prime}=0}^{z-1}\left(1-\frac{M}{N+z^{\prime}+1}\right) .
$$

Thus, rather than identifying all the new subgraphs and calling the reservoir algorithm for each, we can keep a skip counter $Z_{R S}$ that is distributed with the probability mass function given in Eq. (1), and compute its value in constant time using Vitter's acceptancerejection algorithm [35]. Then, based on the value of $Z_{R S}$ that denotes the number of new subgraph insertions we can safely skip, we can decide on the fly whether we should insert into the sample any of the new subgraphs created due to the insertion of edge $(u, v)$. Then, based on the value of $Z_{R S}$ that denotes the number of subgraph insertions we can safely skip, we can decide on the fly whether and how many of the new subgraphs among the $\mathcal{W}$ newly created ones we should select into the sample. Given that a new $k$-subgraph $G_{S}=(S, E(S))$ can be formed only when $E(S) \backslash(u, v)$ is not already an induced subgraph, we can compute the exact value of $\mathcal{W}$ as in Algorithm 3. The pseudocode of the optimized algorithm for incremental streams is given in Algorithm 4.

A similar optimization to avoid the processing of each newly created subgraph in $H \subseteq N_{u, h}^{t-1} \cup N_{v, j}^{t-1}$, for all admissible values of $h, j>0$, is also possible for fully-dynamic streams with proper adjustment of the skip counter based on the value $d=c_{1}+c_{2}$ of uncompensated deletions. Recall that when $d=0$, reservoir sampling is effective for maintaining the uniformity of the sample, hence, we can compute the value of the skip counter $Z_{R S}$ as in the case of incremental streams. When $d>0$, the random-pairing step is effective, for which we exploit Vitter's acceptance-rejection algorithm for list-sequential sampling as follows.

Let $Z_{R P}$ be the random variable that denotes the number of new subgraphs that are not accepted into the sample after the last time a subgraph was deleted (not necessarily from the sample) due to the deletion of an edge. Assume without loss of generality that the deletion of a subgraph was followed by the creation of $d$ new subgraphs due to at least one edge insertion. Following the fact that the new elements that random pairing includes into the sample form a uniform random sample of size $c_{1}$ among $d$ new elements [13], the probability that the random pairing will not accept the next $z$ new subgraphs in $\mathcal{S}$ is given by:

$$
\operatorname{Pr}\left[Z_{R P}=z\right]=\frac{c_{1}}{d-z} \prod_{z^{\prime}=0}^{z-1}\left(1-\frac{c_{1}}{d-z^{\prime}}\right) .
$$

Thus, after each edge deletion, we can compute in constant time the value of skip counter $Z_{R P}$ for random pairing using Vitter's acceptance-rejection algorithm [34] for list-sequential sampling and decide on the fly whether and how many we should insert into the sample any of the new $\mathcal{W}$ subgraphs created in the pairing step. The algorithm to compute the exact number $\mathcal{D}$ of deleted induced subgraphs when an edge $(u, v)$ is deleted at time $t$ is similar to the computation of $\mathcal{W}$, but operates on the neighborhoods at time $t$ instead of time $t-1$ (omitted due to space constraints). The pseudocode of the optimized algorithm is given in Algorithm 5.

\subsection{Derivation for sample size}

Now we provide a lower bound on the size of the sample $\mathcal{S}$ such that $\tilde{\mathcal{F}}_{\tau}^{k}(\epsilon, \delta)$ computed on $\mathcal{S}$ provides an $(\epsilon, \delta)$-approximation to $\mathcal{F}_{\tau}^{k}$.

Lemma 3.1. Suppose that $|\mathcal{S}|=M$ satisfies

$$
M \geq \log \left(\frac{T_{k}}{\delta}\right) \cdot \frac{(4+\epsilon)}{\epsilon^{2}}
$$

Then, for any isomorphism class $i \in\left[1, T_{k}\right]$, the following inequality holds with probability at least $1-\delta / T_{k}$ :

$$
\left|\hat{p_{i}}-p_{i}\right| \leq \epsilon / 2
$$

Proof. Let $X_{i}$ denote an indicator random variable that equals 1 if a randomly sampled subgraph $G_{S}$ from $C^{k}$ belongs in $C_{i}^{k}$ and 0 otherwise, $\forall i \in\left[1, T_{k}\right]$. Notice that $X_{i} \sim \operatorname{Bernoulli}\left(p_{i}\right)$. W.l.o.g, let $G_{j}, j \in[1, M]$ denote the $j$-th subgraph in $\mathcal{S}$ for an arbitrary ordering of the subgraph and let $X_{i}^{1}, \cdots, X_{i}^{M}$ be iid copies of $X_{i}$ where each $X_{i}^{j}$ denotes the event $\mathbb{1}_{\left[G_{j} \in C_{i}^{k}\right]}$.

Using the two-sided Chernoff bounds we have

$$
\operatorname{Pr}\left(\left|\sum_{j=1}^{M} X_{i}^{j}-p_{i} M\right| \geq \theta M p_{i}\right) \leq 2 \exp \left(-\frac{\theta^{2}}{2+\theta} \cdot p_{i} M\right),
$$




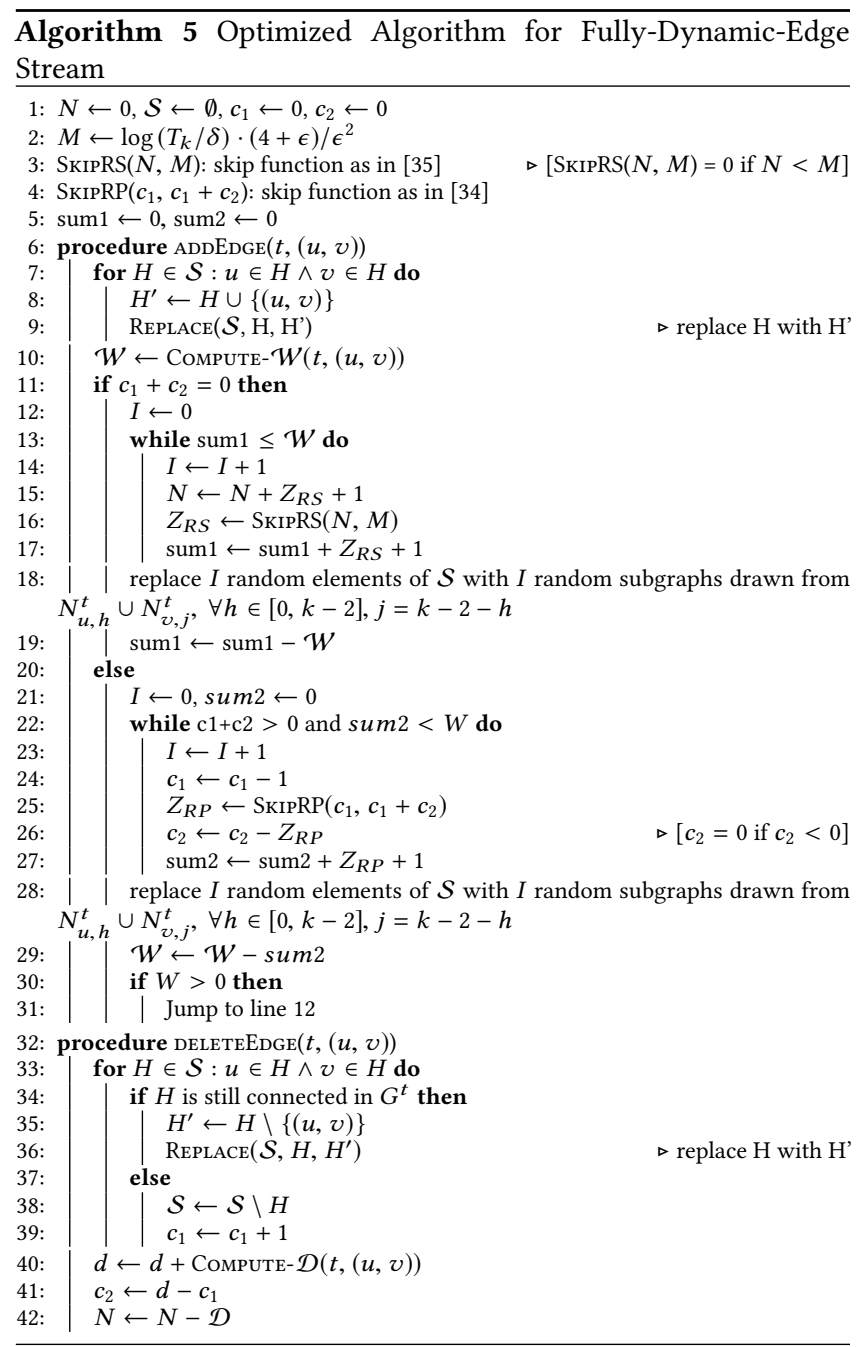

which implies

$$
\operatorname{Pr}\left(\left|\hat{p}_{i}-p_{i}\right| \geq \theta p_{i}\right) \leq 2 \exp \left(-\frac{\theta^{2}}{2+\theta} \cdot p_{i} M\right) .
$$

Here, the two events inside the $\operatorname{Pr}(\cdot)$ are the same events, just divided by $M$ hence the probability remains unchanged. Now, let $\epsilon=2 p_{i} \theta$. Substituting $\theta=\epsilon /\left(2 p_{i}\right)$ we have

$$
\operatorname{Pr}\left(\left|\hat{p}_{i}-p_{i}\right| \geq \epsilon / 2\right) \leq 2 \exp \left(-\frac{\epsilon^{2} / 4}{2 p_{i}+\epsilon / 2} \cdot M\right) .
$$

To obtain a failure probability of at most $\delta / T_{k}$, we would like to have for each $i \in\left[1, T_{k}\right]$

$$
2 \exp \left(-\frac{\epsilon^{2} / 4}{2 p_{i}+\epsilon / 2} \cdot M\right) \leq \frac{\delta}{T_{k}} .
$$

Rearranging the terms we have

$$
M \geq \log \left(\frac{T_{k}}{\delta}\right) \cdot \frac{2 p_{i}+\epsilon / 2}{\left(\epsilon^{2} / 2\right)} .
$$

$T_{k}$ As we want this to hold $\forall i \in\left[1, T_{k}\right]$, we want to have

$$
M \geq \log \left(\frac{T_{k}}{\delta}\right) \cdot \frac{2 p_{\max }+\epsilon / 2}{\left(\epsilon^{2} / 2\right)},
$$

where $p_{\max }=\underset{i \in\left[1, T_{k}\right]}{\max } p_{i}$. Using the worst-case $p_{\max }=1$, we have

$$
M \geq \log \left(\frac{T_{k}}{\delta}\right) \cdot \frac{(4+\epsilon)}{\epsilon^{2}} .
$$

Theorem 3.2. Given a uniform sample $\mathcal{S} \subseteq C^{k}$ of size $M$ that satisfies Eq. (3), $\tilde{\mathcal{F}}_{\tau}^{k}(\epsilon, \delta)$ provides $(\epsilon, \delta)$-approximation to $\mathcal{F}_{\tau}^{k}$.

Proof. Given $M$ that satisfies Eq. (3), using union bound over all $T_{k}$ estimation failure scenarios, we have $\left|\hat{p_{i}}-p_{i}\right| \leq \epsilon / 2$, for all $i \in\left[1, T_{k}\right]$, with probability at least $1-\delta$. Then, there should be no $i \in\left[1, T_{k}\right]$ with $p_{i} \geq \tau$, for which $\hat{p}_{i}<\tau-\epsilon / 2$. Hence, we ensure $\tilde{F}(\mathcal{S}, \tau-\epsilon / 2) \subseteq F\left(C^{k}, \tau\right)$ with probability at least $1-\delta$. Now, assume that there is a subgraph $G_{S} \in C_{i}^{k}$ such that $p_{i}<\tau-\epsilon$. We have that $\hat{p}_{i}<\tau-\epsilon / 2$, hence, there is no subgraph $G_{S}$ such that $G_{S} \notin F\left(C^{k}, \tau\right)$ and $G_{S} \in \tilde{F}(\mathcal{S}, \tau-\epsilon / 2)$, with probability at least $1-\delta$

\section{NEIGHBORHOOD EXPLORATION}

The skip optimizations allows us to efficiently maintain the uniformity of the sample $\mathcal{S}$ by eliminating the need to test the inclusion of each newly created $k$-subgraph in the local neighborhood of the inserted edge. However, the skip optimizations require to know the number $\mathcal{W}$ of new $k$-subgraphs. Unfortunately, exact computation of $\mathcal{W}$ requires costly traversal of the neighborhood of the inserted edge. Moreover, for dynamic streams, the value of the skip counter directly depends on $c_{1}$ and $c_{2}$, which require to compute the number $\mathcal{D}$ of deleted induced subgraphs after each edge deletion operation. Thus, we resort on efficient methods to approximate the values of $\mathcal{W}$ and $\mathcal{D}$.

To efficiently approximate the value of $\mathcal{W}$ after an edge $(u, v)$ is inserted at time $t$, we use sketches to estimate $\left|N_{u, h}^{t-1} \cap N_{v, j}^{t-1}\right|$ for all possible values of $h \in[0, k-2]$ and $j \in[0, k-2]$. Similarly, to efficiently approximate $\mathcal{D}$ after an edge $(u, v)$ is deleted at time $t$, we use sketches to estimate $\left|N_{u, h}^{t} \cap N_{v, j}^{t}\right|$ for all possible values of $h \in[0, k-2]$ and $j \in[0, k-2]$.

Any sketching tecnique for set-size estimation can be used. For our purpose, we choose to use the bottom- $k$ sketch [10] in conjunction with recently-proposed improved estimators for union and intersections of sketches [32]. A bottom- $k$ sketch uses a hash function $h(\cdot)$ to map elements of a universe into real numbers in $[0,1]$, and stores the $k$ minimum values in a set. The smaller the $k$-th stored value is, the larger the size of the original set should be; a simple estimate of the size is given by $\frac{k-1}{\gamma}$, where $\gamma$ is the largest stored hash value.

In our case, the universe of elements is the set of vertices $V^{t}$ that belong to the graph at time $t$. We build a sketch for each vertex $v \in V^{t}$ that summarizes $N_{v}^{t}$. These sketches can be efficiently combined to create a sketch for the union of the neighbors of a given vertex while exploring the neighborhood via a breadth first search (BFS).

Bottom- $k$ sketches can easily be built incrementally. When a new edge $(u, v)$ is added, we simply add the hash value of $v$ to the 
sketch of $u$ if it is smaller than the current maximum, and vice versa. Alas, bottom- $k$ sketches do not directly support deletions. However, traditionally the sketches are used in a streaming setting where memory is the main concern. In our case, the universe of elements already resides in memory (i.e., the vertices of the graph), and our goal is to improve the speed of computation of Algorithm 3 and its counterpart for deletion. Therefore, we can easily store the global hash value of each vertex to be used for sketching. Then, we can implement the sketch by using a pair of min-heap/max-heap. The max-heap $A^{+}$has bounded size and contains the hash values of the corresponding bottom- $k$ vertices. The min-heap $A^{-}$contains the hash values of the rest of the neighborhood. Whenever an edge $(u, v)$ is deleted, if $h(v) \in A^{-}$we remove the value from $A^{-}$but the sketch remains unchanged; if $h(v) \in A^{+}$we remove the value from $A^{+}$, and we also transfer the minimum value from $A^{-}$to $A^{+}$ to maintain the fixed size of the sketch.

\subsection{Efficient implementation of $\mathcal{S}$}

The reservoir sample $\mathcal{S}$ needs to support two main access operations efficiently: (1) Random access (to replace subgraphs in the sample, for reservoir sampling); (2) Access by vertex id (to identify modified subgraphs, as in Algorithm 4).

In order to support both operations in constant time, we resort to an array for the basic random access, supplemented by hash-based indexes for the access by vertex id.

The basic array is straightforward to implement, as the size of the sample $M$ is fixed, and the size its element is constant $k^{2}$ (to store both vertices and edges). On top of this basic array, we maintain and index $\mathcal{I}: V \rightarrow\{S \subset V\}$ such that $v \rightarrow S$ for all $v \in S$ and all $S \in \mathcal{S}$ That is, we have a pointer from each vertex part of a subgraph in the sample, to the set of subgraphs containing it. Therefore, when an edge $(u, v)$ is modified at time $t$ (either added or deleted), retrieving the set of potentially affected subgraphs takes constant time. For each potentially affected subgraph, checking whether it is actually affected also takes constant time: for a subgraph $S \in \mathcal{I}(u)$ (respectively, $S \in \mathcal{I}(v)$ ) we simply need to check whether $v \in S$ (respectively, $u \in S$ ). If so, the subgraph needs to be updated, and so the corresponding counters for its pattern.

\section{EXPERIMENTS}

We conduct an extensive empirical evaluation of the proposed algorithms, and provide a comparison with the existing solutions. In particular, we answer the following interesting questions:

Q1: What is the quality of frequent patterns for incremental streams? Q2: What is the quality of frequent patterns for dynamic streams? Q3: What is the performance in terms of average update time?

\subsection{Experimental setup}

Datasets. Table 1 shows the graphs used as input in our experiments. All datasets used are publicly available. Patent (PT) [14] contains citations among US Patents from January 1963 to December 1999; the label of a patent is the year it was granted. YouTube (YT) [9] lists crawled videos and their related videos posted from February 2007 to July 2008. The label is a combination of a video's rating and length. The streams are generated by permuting the edges in a random order.
Table 1: Datasets used in the experiments.

\begin{tabular}{llrrr}
\hline Dataset & Symbol & $|V|$ & $|E|$ & $|L|$ \\
\hline Patents & PT & $3 \mathrm{M}$ & $14 \mathrm{M}$ & 37 \\
Youtube & YT & $4.6 \mathrm{M}$ & $43 \mathrm{M}$ & 108 \\
\hline
\end{tabular}

Metrics. We use the following metrics to evaluate the quality of all the algorithms:

- Average Relative Error (RE): measures how close the estimation of the frequency of the subgraph patterns compared to the ground truth. For the set of patterns $\mathcal{F}_{\tau}^{k}$, the average RE of the estimation is defined as $\frac{1}{T_{k}} \sum_{i=1}^{T_{k}} \frac{\left|\hat{p}_{i}-p_{i}\right|}{p_{i}}$.

- Precision: measures the fraction of frequent subgraph patterns among the ones returned by the algorithm.

- Recall: measures the fraction of frequent subgraph patterns returned by the algorithm over all frequent subgraphs (as computed by the exact algorithm).

Additionally, we evaluate the efficiency of the algorithms by reporting the average update time. We provide an extensive comparison of all the algorithms for $k=3$. We report the results of experiments averaged over 5 runs.

Algorithms. We use two baselines. Exact counting (EC) performs exhaustive exploration of the neighborhood of the updated edge, and counts all possible subgraph patterns. Edge reservoir (ER) is a scheme inspired by Stefani et al. [31], which maintains a reservoir of edges during the dynamic edge updates. The edge reservoir is used to estimate the frequency of subgraph patterns by applying the appropriate correcting factor for the sampling probability of each pattern. We compare these baselines with our proposed algorithms, subgraph reservoir (SR) and its optimized version (OSR). The size of the subgraphs reservoir is set as in Section 3.4. Unless otherwise specified, we fix $\epsilon=0.01$ and $\delta=0.1$. To have a fair comparison with ER, following the evaluation of Stefani et al. [31], we set the size of edge reservoir as the maximum number of edges used in the subgraph reservoir, averaged over 5 runs.

Experimental environment. We conduct our experiments on a machine with 2 Intel Xeon Processors E5-2698 and 128GiB of memory. All the algorithms are implemented in Java and executed on JRE 7 running on Linux. The source code is available online. ${ }^{3}$

\subsection{Incremental case}

We first evaluate our proposed algorithm on incremental streams. Starting from an empty graph, we add one edge per timestamp, for both the PT and YT datasets, and run the algorithms for several values of the frequency threshold $\tau$.

Figure 1 shows the results. For the PT dataset, the three algorithms behave similarly in terms of RE. The subgraph versions offer slightly higher precision at the expense of decrease in recall. However, for the highest frequency threshold, we see a marked deterioration of the performance of ER. This behavior is a result of higher variance in ER due to non-uniform subgraph-sampling probabilities. Conversely, for YT, both versions of the subgraph reservoir algorithm provide superior results in terms of average relative error. Considering YT is the larger and more challenging

$\overline{{ }^{3} \text { https://github.com/anisnasir/frequent-patterns }}$ 

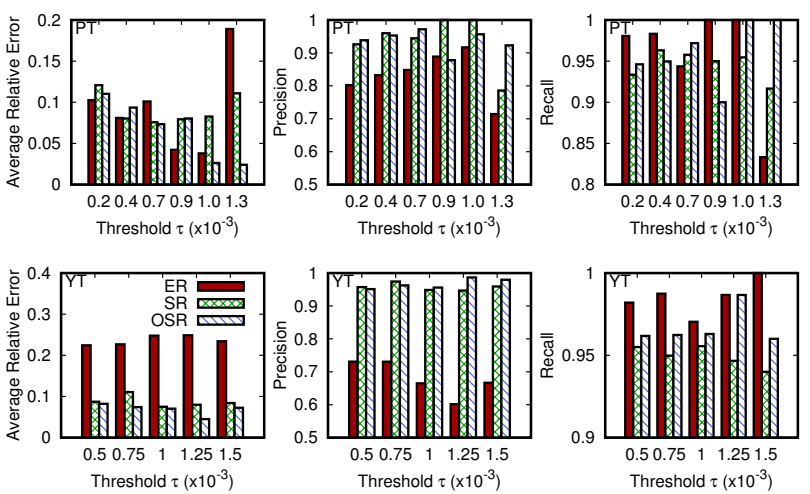

Figure 1: Relative error, precision, and recall for incremental streams on PT and YT datasets, for different values of threshold $\tau$.

dataset (in terms of number of labels), this result shows the power of subgraph sampling. The improved estimation performance translates to much higher precision for SR and OSR compared to ER. The recall of all the algorithms are very similar. Overall, the results indicate that ER generates a larger number of false positives in the result set, while SR and OSR are able to avoid such errors while at the same time still having a low false-negative rate.

\subsection{Fully-dynamic case}

Now, we proceed to evaluate the algorithms for fully-dynamic streams. To produce edge deletions, we execute the algorithms in a sliding window model. This model is of practical interest as it allows to observe recent trends in the stream. We evaluate the algorithms for the YT dataset, and use a sliding window of size $10 \mathrm{M}$. We choose a sliding window large enough so so that the number of edges (subgraphs) do not fit in the edge (subgraph) reservoir, otherwise both algorithms are equivalent to exact counting. We only report the results for YT dataset, as the result for the PT dataset are similar to the incremental case.

Figure 2 contains the results for YT dataset. ER obtains higher relative error compared to SR, and poor precision and recall. SR is clearly the best performing algorithm in terms of accuracy, however, as we shown next, it pays in terms of efficiency. OSR has consistently better accuracy than ER, although the approximations it deploys introduce some errors. This effect is more evident for larger frequency thresholds, where the precision drops noticeably.

\subsection{Performance}

Lastly, we evaluate the algorithms in terms of the average update time for both incremental and fully-dynamic streams on PT and YT datasets. The size of the sliding window is $10 \mathrm{M}$ for the fullydynamic streams. Figure 3 reports the results of the experiments which show that both SR and OSR provide significant performance gains compared to the EC while they are both outperformed by ER However, given the superior accuracy of SR and OSR compared to ER, it can be easily observed that OSR provides a good trade-off between accuracy and efficiency.
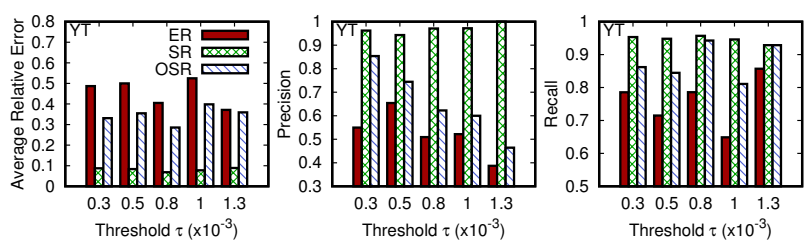

Figure 2: Relative error, precision, and recall for fullydynamic stream on YT dataset, for different values of threshold $\tau$.

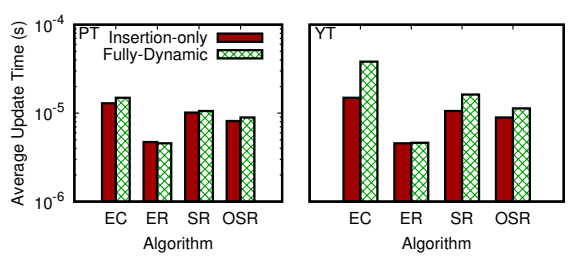

Figure 3: Average update time for incremental and fullydynamic streams on PT and YT datasets.

\section{RELATED WORK}

Triangle counting. Exact and approximate triangle counting in static graphs has attracted a great deal of attention. We refer the reader to the survey by Latapy [25] for a comprehensive treatment of the topic, and include here only work on approximate triangle counting in incremental or fully-dynamic streams. Tsourakakis et al. [33] proposed triangle sparsifiers to approximate the triangle counts with a single pass of the graph, hence, the technique can also be applied to incremental streams. Pavan et al. [28] and Jha et al. [19] proposed sampling a set of connected paths of length for approximately counting the triangles in incremental streams. Lim and Kang [26] proposed an algorithm based on Bernoulli sampling of edges for incremental streams, in which the edges are kept in the sample with a fixed user-defined probability. Recently, Stefani et al. [31] proposed an algorithm for fully-dynamic streams via reservoir sampling [35] and random pairing [12].

General $k$-vertex graphlet counting. Approximate counting of 3-, 4-, 5-vertex graphlets in static graphs has received much more attention than exact counting, which has an exponential cost. Most of the literature on approximate counting of graphlets uses randomwalks to collect a uniform sample of graphlets on static graphs [2, $7,15,36]$. Alternatively, Bressan et al. [4] proposed a color coding based scheme for estimating $k$-vertex graphlet statistics. Unlike the static case, approximating graphlet statistics in a streaming setting has received much less attention, and the literature is limited to incremental streams for $k>3$. Wang et al. [37] are the first to propose an algorithm that estimates graphlet statistics from a uniform sample of edges in incremental streams. A recent work by Chen and Lui [8] examines approximate counting of graphlets in incremental streams for different choice of edge sampling and probabilistic counting methods. 
Transactional FSM. Inokuchi et al. [18] introduced the problem of FSM in the transactional setting, where the goal is to mine all the frequent subgraphs on a given dataset of many, usually small, graphs. Following this seminal work, a good number of algorithms for FSM task in transactional setting were provided [17, 22, 27, 38]. The transactional FSM setting is similar to the one considered by frequent-itemset mining [16], hence, many existing results can be reused thanks to the anti-monotonicity of the support metric (i.e., count of isomorphisms). In addition to the exact mining approaches, which do not scale to large graph datasets [5], a line of work has studied the approximate mining of frequent subgraphs by MCMC sampling from the space of graph patterns $[1,30]$ with efficient pruning strategies based on anti-monotonicity of the support metric. For a comprehensive treatment, see the survey by Jiang et al. [20].

Single-Graph FSM. Kuramochi and Karypis [24] proposed an algorithm for exact mining of all frequent subgraphs in a given static graph that enumerates all the isomorphisms of the given graph and relies on the maximum-independent set (MIS) metric whose computation is NP-Complete. Elseidy et al. [11] proposed an apriori-like algorithm for exact mining of all frequent subgraphs based on the MIS metric from a given static graph. Apart from the exact mining algorithms, a line of work focused on approximate mining of frequent subgraphs in a given static graph. Kuramochi and Karypis [23] proposed a heuristic approach that prunes largely the search space however discovers only a small subset of frequent subgraphs without provable guarantees. Chen et al. [6] uses an approximate version of the MIS metric, allowing approximate matches during the pruning. Khan et al. [21] propose proximity patterns, which, by relaxing the connectivity constraint of subgraphs, identify frequent patterns that cannot be found by other approaches.

While the discussed work for solving the FSM problem on a given static graph are promising, none of them are applicable to streaming graphs. The closest to our setting is the work by Ray et al. [29] which consider a single graph with continuous updates, however their approach is a simple heuristic applicable only to incremental streams and without provable guarantees.

\section{CONCLUSION}

We initiated the study of approximate frequent-subgraph mining (FSM) in both incremental and fully-dynamic streaming settings, where the edges can be arbitrarily added or removed from the graph. For each streaming setting, we proposed algorithms that can extract a high-quality approximation of the frequent $k$-vertex subgraph patterns, for a given threshold, at any given time instance, with high probability. Our algorithms operate by maintaining a uniform sample of $k$-vertex subgraphs at any time instance, for which we provide theoretical guarantees. We also proposed several optimizations to our algorithms that allow achieving high accuracy with improved execution time. We showed empirically that the proposed algorithms generates high-quality results compared to natural baselines.

\section{REFERENCES}

[1] Mohammad Al Hasan and Mohammed J Zaki. 2009. Output space sampling for graph patterns. PVLDB 2, 1 (2009), 730-741.

[2] Mansurul A Bhuiyan, Mahmudur Rahman, and M Al Hasan. 2012. Guise: Uniform sampling of graphlets for large graph analysis. In ICDM. 91-100.

[3] Albert Bifet, Geoff Holmes, Bernhard Pfahringer, and Ricard Gavaldà. 2011 Mining frequent closed graphs on evolving data streams. In KDD '11. 591-599.
[4] Marco Bressan, Flavio Chierichetti, Ravi Kumar, Stefano Leucci, and Alessandro Panconesi. 2017. Counting Graphlets: Space vs Time. In WSDM. 557-566.

[5] Vineet Chaoji, Mohammad Al Hasan, Saeed Salem, Jeremy Besson, and Mohammed J Zaki. 2008. Origami: A novel and effective approach for mining representative orthogonal graph patterns. The ASA Data Science fournal 2008 1, 2 (2008), 67-84.

[6] Chen Chen, Xifeng Yan, Feida Zhu, and Jiawei Han. 2007. gapprox: Mining frequent approximate patterns from a massive network. In ICDM.

[7] Xiaowei Chen, Yongkun Li, Pinghui Wang, and John Lui. 2016. A general framework for estimating graphlet statistics via random walk. PVLDB 10, 3 (2016), 253-264.

[8] Xiaowei Chen and John Lui. 2017. A unified framework to estimate global and local graphlet counts for streaming graphs. In ASONAM. 131-138.

[9] Xu Cheng, Cameron Dale, and Jiangchuan Liu. 2008. Statistics and social network of youtube videos. In IWQOS. 229-238.

[10] Edith Cohen and Haim Kaplan. 2007. Summarizing data using bottom-k sketches. In PODC. 225-234.

[11] Mohammed Elseidy, Ehab Abdelhamid, Spiros Skiadopoulos, and Panos Kalnis. 2014. Grami: Frequent subgraph and pattern mining in a single large graph. PVLDB 7, 7 (2014), 517-528.

[12] Rainer Gemulla, Wolfgang Lehner, and Peter J Haas. 2006. A dip in the reservoir: Maintaining sample synopses of evolving datasets. In PVLDB. 595-606.

[13] Rainer Gemulla, Wolfgang Lehner, and Peter J Haas. 2008. Maintaining boundedsize sample synopses of evolving datasets. VLDBf 17, 2 (2008), 173-201.

[14] Bronwyn H Hall, Adam B Jaffe, and Manuel Trajtenberg. 2001. The NBER patent citation data file: Lessons, insights and methodological tools. Technical Report. National Bureau of Economic Research.

[15] Guyue Han and Harish Sethu. 2016. Waddling random walk: Fast and accurate sampling of motif statistics in large graphs. arXiv:1605.09776 (2016)

[16] Jiawei Han, Jian Pei, and Micheline Kamber. 2011. Data mining: concepts and techniques. Elsevier.

[17] Jun Huan, Wei Wang, and Jan Prins. 2003. Efficient mining of frequent subgraphs in the presence of isomorphism. In ICDM.

[18] Akihiro Inokuchi, Takashi Washio, and Hiroshi Motoda. 2000. An apriori-based algorithm for mining frequent substructures from graph data. In ECML-PKDD.

[19] Madhav Jha, C Seshadhri, and Ali Pinar. 2015. A space-efficient streaming algorithm for estimating transitivity and triangle counts using the birthday paradox. TKDD (2015).

[20] Chuntao Jiang, Frans Coenen, and Michele Zito. 2013. A survey of frequent subgraph mining algorithms. The Knowledge Eng. Review 28, 1 (2013), 75-105.

[21] Arijit Khan, Xifeng Yan, and Kun-Lung Wu. 2010. Towards proximity pattern mining in large graphs. In SIGMOD. 867-878.

[22] Michihiro Kuramochi and George Karypis. 2001. Frequent subgraph discovery. In ICDM. 313-320.

[23] Michihiro Kuramochi and George Karypis. 2004. Grew-a scalable frequent subgraph discovery algorithm. In ICDM.

[24] Michihiro Kuramochi and George Karypis. 2005. Finding frequent patterns in a large sparse graph. Data mining and knowledge discovery 11, 3 (2005), 243-271.

[25] Matthieu Latapy. 2008. Main-memory triangle computations for very large (sparse (power-law)) graphs. Theoretical Comp. Sci. 407, 1-3 (2008), 458-473.

[26] Yongsub Lim and U Kang. 2015. Mascot: Memory-efficient and accurate sampling for counting local triangles in graph streams. In KDD. 685-694.

[27] Siegfried Nijssen and Joost N Kok. 2004. A quickstart in frequent structure mining can make a difference. In $K D D$.

[28] Aduri Pavan, Kanat Tangwongsan, Srikanta Tirthapura, and Kun-Lung Wu. 2013. Counting and sampling triangles from a graph stream. PVLDB (2013).

[29] Abhik Ray, Larry Holder, and Sutanay Choudhury. 2014. Frequent Subgraph Discovery in Large Attributed Streaming Graphs. In BigMine. 166-181.

[30] Tanay Kumar Saha and Mohammad Al Hasan. 2015. FS3: A sampling based method for top-k frequent subgraph mining. Statistical Analysis and Data Mining: The ASA Data Science fournal 8, 4 (2015), 245-261.

[31] Lorenzo De Stefani, Alessandro Epasto, Matteo Riondato, and Eli Upfal. 2017. Trièst: Counting local and global triangles in fully dynamic streams with fixed memory size. TKDD 11, 4 (2017), 43

[32] Daniel Ting. 2016. Towards optimal cardinality estimation of unions and intersections with sketches. In KDD '16. 1195-1204.

[33] Charalampos E Tsourakakis, Mihail N Kolountzakis, and Gary L Miller. 2011. Triangle Sparsifiers. f. Graph Algorithms Appl. 15, 6 (2011), 703-726.

[34] Jeffrey Scott Vitter. 1984. Faster methods for random sampling. Commun. ACM 27, 7 (1984), 703-718.

[35] Jeffrey S Vitter. 1985. Random sampling with a reservoir. TOMS 11, 1 (1985), 37-57.

[36] Pinghui Wang, John Lui, Bruno Ribeiro, Don Towsley, Junzhou Zhao, and Xiaohong Guan. 2014. Efficiently estimating motif statistics of large networks. TKDD 9, 2 (2014), 8.

[37] Pinghui Wang, John CS Lui, Don Towsley, and Junzhou Zhao. 2016. Minfer: A method of inferring motif statistics from sampled edges. In ICDE.

[38] Xifeng Yan and Jiawei Han. 2002. gSpan: Graph-Based Substructure Pattern Mining. In ICDM. 721-724. 